\title{
$\underline{\text { On-line Compensation in a Digital Coriolis Mass Flow Meter }}$
}

\author{
Manus Henry \\ Department of Engineering Science, Oxford University.
}

\begin{abstract}
$\underline{\text { Abstract }}$
For many years it has been demonstrated that the use of digital technology, particularly embedded microprocessors, can improve flowmeter performance in various ways (e.g. temperature compensation, elimination of drift, generation of engineering units, diagnostics). A self-validating or SEVA sensor uses in-built processing power to generate generic metrics of measurement quality, based on on-line uncertainty. This uncertainty includes all factors effecting the on-line measurement, including diagnostics but also manufacturing aspects such as the components used and the characterisation procedure. This paper describes a prototype SEVA coriolis mass flow meter transmitter built primarily from digital components, which provides compensation for both faults and manufacturing limitations. A technique to compensate for drift and imbalance in the transmitter front-end circuitry is described in detail. The prototype has been developed using a hardware/software co-design approach in which virtually all aspects of instrument design are described in software, but which can be implemented flexibly in either hardware or software according to economic requirements. This approach offers manufacturers the opportunity of incorporating the latest components into their products rapidly in order to remain competitive.
\end{abstract}

\section{$\underline{\text { Keywords }}$}

Sensor Validation. Coriolis Mass Flow Metering. Digital Technology. Hardware/software co-design. 


\section{Nomenclature}

$A_{i}$

amp_factor

Diff

$G_{n}$

$G_{p}$

I

Ratio

Sum

$S V(t)$

$t_{Z_{o}}$

$Z_{o}$

$\varepsilon_{G}$

$\varphi_{Z_{o}}$

$\omega$

Subscripts

1,2

C

est

$\mathrm{N}$

$\mathrm{P}$

S amplitude of ith harmonic in sensor signal (V)

amplitude factor

difference function

gain of negative portion of sensor signal

gain of positive portion of sensor signal

Integral function

Ratio function

Sum function

sensor voltage signal (V)

time delay of zero crossing due to voltage offset $\mathrm{Z}_{\mathrm{o}}(\mathrm{s})$

voltage offset $(\mathrm{V})$

gain imbalance factor

phase delay due to voltage offset $Z_{0}(s)$

frequency of oscillation ( $\mathrm{rad} / \mathrm{s})$

first or second harmonic

using a cosine function multiplier

estimating function

negative sensor voltage section

positive sensor voltage section

using a sine function multiplier 


\section{Introduction}

The control and instrumentation sector of industry is undergoing a major transformation in response to advances in technology. At least three aspects of the role of the instrument are changing:

- The functionality embedded in each device is being extended (for example by the provision of self-calibration, maintenance and operational history, and internal diagnostics), in response to the availability of embedded processing power. In the case of certain instruments, such as coriolis mass flow meters, there are also rapid developments in fundamental research (e.g. Cheesewright and Clark, 1998, Cheesewright et al., 2000).

- The relationship between the instrument and the system is being transformed (for example in the distribution of control decisions to local nodes) by the introduction of digital communication standards, in particular Fieldbus (Henry, 1995b).

- The technology used to implement commercial instruments is undergoing rapid evolution, with new components (e.g. sigma-delta converters, memory and processors) offering continuous improvements in price/specification and, critically, power consumption.

In the past, technology limitations allowed only simple models of instrument behaviour (e.g. the 4-20mA standard for measurement communication). Such models provided stability for both users and manufacturers over many years. However, the new technologies allow many alternative strategies for improving performance. Extra functionality is now being provided in today's instruments, but at the expense of consensus and inter-operability, as witnessed particularly by the fieldbus communications standards debate.

If users face difficult purchasing decisions, manufacturers face far more difficult developmental choices. It is already clear that users will expect improvements (as measured by enhanced plant efficiency and safety, and reduced operational and maintenance costs), at a minimal or zero price premium (Henry, 2000). In addition, with the trend for reducing engineering staff and purchasing turnkey solutions, it is important that operators are shielded from the full complexity of the new technology. Manufacturers must choose what functionality to place inside their devices, and what communication standards to adopt, but they must also choose implementation technology. A wrong technology choice may result in an otherwise excellent product being undercut in the market, or being dependent on components that are no longer manufactured or supported. There is thus increasing interest in cutting down the development cycle, maximising re-use of design effort, and taking final 
decisions about implementation technology as late as possible. Like the instruments themselves, the techniques and tools of design and implementation must be subject to a ruthless process of continuous improvement.

The SEVA Research Group at Oxford began in 1988 to examine the impact of the new technology on instruments, in particular with regard to local fault detection. It developed a theoretical model of how a 'self-validating' or SEVA instrument should behave. The SEVA sensor model (Henry and Clarke, 1993) is now being considered as a UK national standard for the reporting of measurement quality in industrial sensors (SIRA, 1998; Wood, 2000). It assumes the availability of internal computing power for self-diagnostics based on the designer's expertise, and of digital communications to convey measurement, diagnostic and maintenance data. The SEVA model proposes a generic set of metrics for describing measurement quality. The key metric is the on-line uncertainty of each measurement. This permits generic strategies for responding to changes in measurement quality at the control system level.

The on-line uncertainty takes into account all error sources affecting the on-line measurement, including:

- The transduction itself - the mapping from the true process measurand to the observed transducer signal.

- The components used to manufacture the instrument (based for example upon manufacturer's spec sheets, but confirmed via laboratory testing of assembled circuit boards);

- The characterisation procedure at the end of the production line (accuracy and stability of the reference conditions and data reduction effects, resulting in uncertainty values for each of the characterisation coefficients);

- The effect of any faults, whether instrument- or process-induced, after compensation has been applied.

An example of a SEVA analysis of a thermocouple, in which measurement errors due to component, environment and sensor fault effects are considered, is given by Yang and Clarke (1997). The appeal of the SEVA approach is that it proposes an on-line, dynamic estimate of measurement quality, establishing traceability from design, through production and characterisation, to the individual measurement in-situ. For the manufacturer, any improvement in the production process (for example increasing the accuracy of the characterisation references, or the use of higher specification components) can be reported to 
the user via a reduction in the uncertainty of the on-line measurement. For the user, on-line uncertainty can be independently verified via laboratory trials. For both manufacturers and users, on-line uncertainty can be used as a management tool for continuous quality monitoring and improvement.

An important aspect of the Oxford research is the creation of SEVA prototypes based upon commercial devices. For example, the first generation SEVA prototype of a Coriolis mass flow meter consisted of the flowtube and conventional commercial transmitter connected to a PC (Henry, 1995a). The PC and the modified transmitter together showed how a SEVA device would behave. The prototype demonstrated an ability to detect and correct for several fault modes, as well as the generation of SEVA metrics. In practice, it was not viable to incorporate the validation functionality within the existing commercial transmitter. However, one of the benefits of carrying out a sensor validation analysis is that it leads to fundamental redesign (Figure 1). The identification of the major limitations and fault modes of an instrument, and their impact on measurement quality, provides strong motivation for improvements in the basic design (or indeed manufacturing, installation, characterisation, and/or operating procedures where weaknesses are revealed). The idea of an all-digital transmitter design emerged after this first round of validation activity. The primary intention was to replace all the analogue circuitry (other than essential front-end op-amps) with a small number of digital components, and to carry out almost all functionality within software (Henry et al., 2000).

An all-digital design is attractive not only for research purposes, but also for commercial implementation. Audio technology is available for mainstream consumer markets such as mobile phones in large volumes at low per unit cost. The commercial appeal is clear: a low component count and a high software content, based on rapidly evolving, competitive, mainstream technology. However, prototype development raises several of the same issues facing manufacturers, as described above. The choice of implementation technology is important, as it has a strong influence on the quality of validation that can be carried out. On the other hand, it is impossible for a research group to keep up with the continuous improvements in component performance offered by the market. The Valcard research programmes at Oxford (Henry, 1995c; Henry et al., 1996) have addressed this issue through the use of a recently developed hardware/software co-design approach called hardware compilation: the required functionality is defined in software using software tools and techniques. Research prototypes are implemented using off-the-shelf processors to run software and Field Programmable Gate Arrays (FPGAs) to build hardware. A commercial implementation may use a different partition of functionality into hardware and software, and 
use different technologies (e.g. ASIC, low power processors, ADCs etc), while still largely being automatically - and therefore quickly and cheaply - generated from the original software functional specification, which is the core intellectual property. This approach is intended to shorten the delay from prototype to final product, and offers to manufacturers the possibility of very late decisions over product implementation technology, based on the most recent commercial and technical information. As yet, exploitation has been limited to rapid prototyping, but programmes to develop commercial products incorporating this technology are now under way.

Section 2 provides a summary of the design and capabilities of the digital coriolis mass flow meter transmitter; a more detailed overview is provided by (Henry et al., 2000). Section 3 describes an algorithmic technique employed within the transmitter to compensate for manufacturing limitations associated with front-end gain mismatch and DC offset stability. In the absence of compensation, these effects induce significant flow zero drift in the meter output, and hence might require high specification, high cost circuitry to keep zero drift within acceptable limits. With on-line compensation, less stringent manufacturing specifications can be applied. Section 4 discusses how the hardware/software codesign approach has been used to develop prototypes.

\section{The Digital Coriolis Mass Flow Meter}

A Coriolis Mass Flow meter consist of a vibrating flowtube (fig. 2) in series with the process piping, and an electronic transmitter which maintains flowtube vibration, performs measurement calculation, and transmits measurement data to the control system. One or two driver coils are used to excite oscillation of the flowtube, while two position or velocity sensors provide raw measurement data. The frequency of vibration gives fluid density information while the phase difference between the two sensors, generated by coriolis forces, yields the mass flow rate, typically to an accuracy of $0.2 \%$.

The essential measurement requirements are the calculation of the frequency and amplitude of, and phase difference between, the sensor signals. The mapping to engineering units also requires the flowtube temperature to compensate for variable tube stiffness. Typically in current commercial transmitters, frequency is calculated by observing the time delay between successive positive or negative zero crossings in the data from either sensor. To calculate phase difference, a commonly used technique is the so-called time delay method: the time between a zero crossing on one sensor and a zero crossing of the other is approximately proportional to the phase difference between them (Fuller, 1987). 
The transduction process within the flowtube, being physical, is inevitably subject to a variety of error sources, such as mechanical and electrical non-linearities and noise. In addition, there may be externally-induced sources of measurement error (Cheesewright et al., 2000), such as flow pulsation and two-phase flow. However, given the availability of digital technology for transmitter manufacture, it is becoming reasonable to envisage the noise-free transmitter, i.e. one that introduces negligible additional errors beyond those unavoidably caused by transduction and external factors. This is particularly viable for instruments such as the coriolis meter where power and cost constraints are less severe then for two-wire instrumentation. Furthermore (Henry et al, 2000, Cheesewright and Clark, 1998) it is possible to use digital signal processing to detect and ameliorate the effects of flowtube or external factors, thus extending the useful working range of the instrument as a whole. The SEVA metrics provide a convenient framework for reporting the resulting measurement quality.

In the digital prototype transmitter, the raw data are sampled at high speed and computing power is plentiful, and so there are numerous ways of extracting the key parameters, and several strategies have been compared. However, a Fourier-based technique (Henry et al., 2000), has been found to be very precise, and is the basis of the following results.

\section{Repeatability}

Repeatability results for a $25 \mathrm{~mm}$ flowtube operating in a variety of conditions are shown in Table 1. For a fixed input, standard deviations are given based on 10,000 cycles (corresponding to about 1 minute's operation). Absolute, not percentage, values are shown due to the use of zero phase input in several table rows. The figures suggest that for 'pure' sinewave inputs from the signal generator (and indeed for the empty flowtube) precise phase, frequency and amplitude results are obtained from the digital transmitter. Under flow conditions, the poorer precision can reasonably be attributable to genuine variation in flow together with flowtube and external factors, and so it can be concluded that the resolution of the transmitter itself is unlikely to be a significant source of precision error, particularly in the harsh environment of industrial applications.

Possibly a more useful test of repeatability performance is in terms of a process measurement in engineering units, for which data are shown in Fig. 3 and Table 2. Two meters (25mm and $50 \mathrm{~mm}$ ) were placed in series in a flow rig, and their mass totals compared over 20 consecutive runs. Back-to-back trials such as this, where both meters receive the same start and stop totalisation signals, are effective for assessing repeatability as many other potential error sources (e.g. the repeatability and accuracy of the flow rig) are eliminated. Fig. 3 shows the scatter for the $1 \mathrm{~kg} / \mathrm{s}$ run, which is consistent with a normal distribution with a standard 
deviation of $0.005 \%$. Table 2 shows the statistics of several runs with different flow rates and durations. The mean difference $(25 \mathrm{~mm}-50 \mathrm{~mm})$ is a function of the accuracy of each flowtube, while the standard deviation is related to their repeatability or precision. For the shorter runs the poorer repeatability may be attributable to the timing resolution of the start and stop signals (approx. 1/160s).

\section{Accuracy}

Fig. 4 shows, for the digital transmitter working with a $25 \mathrm{~mm}$ flowtube, the mass-flow error (obtained from batch trials) over the range of the instrument, which has a turndown of 100:1. Ten measurements are provided at each flow rate to give a further indication of repeatability performance, although this includes the repeatability of the entire flowrig (e.g. the flow diverter mechanism), and not only the transmitter as in the previous results. The upper and lower bounds show the (static) uncertainty claimed for the commercial flow meter. Clearly in this case the digital transmitter performs well within this specification. In practice it has been found that the linearity of the reading over the flow range depends upon the particular flowtube, which of course has not been improved by the validation research. Again, the most important conclusion is that the digital transmitter is not a significant source of error. Indeed its high precision might be used to provide an additional correction for any non-linearities observed during flowtube characterisation.

\section{Other performance improvements}

As described by Henry et al. (2000), performance improvements are also noted in the following areas. In each case these amount to the use of digital signal processing techniques to detect and compensate for flowtube limitations or external factors.

- Flowtube oscillation control: a new non-linear oscillation control algorithm has been developed (Clarke, 1999) to extend operation down to $0.3 \%$ of the conventional amplitude, drawing only $0.1 \mathrm{~mA}$ per driver for low power operation.

- Compensation for external vibration. Henry et al (2000) contains data which shows that a correction based upon the relative rate of change of amplitude of the sensor signals can correct for the effects of external vibration at some frequencies.

- Start-up from empty. The commercial transmitter has poor performance in batch applications where the flowtube starts empty. In trials, the digital transmitter demonstrates a hundred-fold improvement.

- Two-phase flow. The digital transmitter can maintain flowtube oscillation even in high damping conditions such as two phase flow. Compensation techniques have been devised 
to correct the flow and density measurements for the effects of two-phase flow, in line with the SEVA philosophy.

\section{Compensation for voltage zero offset and gain imbalance}

Earlier, it was suggested that ideally a digital transmitter should introduce negligible additional errors. This assumes that once data is in the digital domain (and again, assuming plentiful computing power available), the numerical errors associated with algorithmic processing are small compared to those associated with the transduction process and the influence of external factors. In most cases however, transmitters are required to convert analogue transducer signals into some form of digital representation, typically through the use of analogue-to-digital converters, and these can be a significant source of additional error. In this section a detailed description is given of a technique implemented within the digital transmitter, to track and compensate for non-idealities within the front end circuitry, and thus to minimise the errors introduced by the transmitter.

Zero offset may be introduced into a sensor voltage signal by drift in the pre-amplification circuitry and by the audio quality analogue-to-digital converter, which is not designed with DC offset and amplitude stability as high priorities. Slight differences in the pre-amplification gains for positive and negative voltages due to the use of differential circuitry may worsen the zero offset effect (see fig. 5). These effects interact, thus preventing the use of simple algorithms such as running mean to eliminate zero offset alone. The errors vary between converters and transmitters, and with both temperature and component wear.

For example, Figure 6 shows how offset and positive and negative gains vary with chip operating temperature for one such device (the two-channel AD1879 converter). The repeatability of the illustrated trends is poor, and even allowing for temperature compensation based on the trends (requiring expensive device-specific calibration), residual zero offset and positive/negative gain mismatch remain.

If phase is calculated using the time difference between zero crossing points on the two sensor voltages, DC offset (which is of course different for each of the two sensor signals) will lead to phase errors. This effect is illustrated by Figs 7A-7C. Each graph shows the calculated phase offset as measured by the digital transmitter when the true phase offset is zero (i.e. at zero flow).

Fig. 7A shows phase calculated based on whole cycles starting with positive zero-crossings (i.e. a point where voltage passes from negative to positive). The mean value is 0.00627 
degrees. Fig. 7B shows phase calculated starting with negative zero-crossings. The mean value is -0.0109 degrees. Fig. 7C shows phase calculated using all zero-crossings (i.e. every half-cycle), and hence interleaves the data from Figs. 7A and 7B. The average phase $(-0.00234)$ is closer to zero than in Figs. 7A and 7B, but the standard deviation of the signal is about six times higher.

More sophisticated phase measurement techniques, such as those based on Fourier methods, are theoretically immune to DC offset. However, it is desirable to eliminate zero offset even when those techniques are used, as it allows a simpler analysis of the effects of, for example, amplitude modulation on apparent phase and frequency (Henry et al, 2000). In addition, gain mismatch between positive and negative voltages will introduce errors into any measurement technique.

This is illustrated in Figure 8, which shows the long term drift in phase where the true flow rate is zero, calculated simultaneously using the time delay and Fourier methods. Each point represents an average over one minute of live data. For comparison, 4 degrees of phase corresponds to full flow; to maintain 1\% accuracy at the bottom end of a 100:1 turndown, it is desirable to keep zero drift to within roughly 1e-4 degrees. Over several hours, the drift is significant using either method of phase calculation. Thus, even if the meter were zeroed every day, which in many applications would be an excessive maintenance requirement, there may still be unacceptable phase drift. Drift is most rapid during the first two hours. Here several temperature-related factors are probably involved, including transmitter warm-up, and laboratory cool-down at the end of the working day. Other factors contributing to phase zero drift, such as the (temperature-varying) mechanical stress on the flowtube through its connections to the pipework, are associated with the flowtube and/or external mechanical factors, and are not addressed by the technique described in this paper. Similarly, the possible influence of other sensor signal components, such as those described by Cheesewright et al. (2000), are not considered here, and may be the subject of further investigation.

\section{Compensation Technique}

An on-line technique for dealing with voltage offset and gain mismatch has been developed which uses the computational capabilities of the digital transmitter and which does not require a zero flow condition. The technique uses a set of calculations each cycle which, when averaged over a reasonable period (e.g., 10,000 cycles or 1 minute), and excluding regions of major change (e.g. any major disturbance to flowtube oscillation such as the onset of two-phase flow), converge on the desired zero offset and gain mismatch compensations. A mathematical description of the required integral functions and their approximations is given 
in Appendix 1, while Appendix 2 outlines the algorithm for calculating the compensation factors.

Figs. 9 and 10 show results obtained using the compensation procedure. The short-term impact on the time delay phase calculation is illustrated in Fig. 9. This shows consecutive phase estimates from start-up with the correction applied, (compare with Fig. 7). To allow settling time after start-up and after each update of voltage offset and gain imbalance, there are additional delays of 3000 and 1000 cycles respectively, and so estimates are generated at cycles 13,000, 24,000, 35,000 etc. The first two corrections have a visible impact, reducing the large phase offsets substantially. Once good estimates of the voltage offset and gain imbalance terms are established, and the transmitter temperature has stabilised, there are no further substantial changes.

Longer term behaviour for both the Fourier and time delay methods of phase difference calculation are shown in Fig 10. In each case the initial large offset is rapidly corrected, and then the phase offset is kept close to zero (i.e. well within 1e-4) over many hours. The gain and offset terms are also shown in Fig. 10. The significant changes in offset values, occurring after approximately 1 hour and 12 hours, are probably attributable to environmental temperature variations at the end and beginning of the working day. These changes occur without any significant impact on the phase difference value, and demonstrates the dynamic behaviour of the correction technique.

Typical values for $Z_{o}$ and $\varepsilon_{G}$, the zero offset and gain mismatch terms for the digital coriolis meter are $Z_{o}=-7.9 \mathrm{e}^{-4}$ and $\varepsilon_{G}=6.9 \mathrm{e}^{-4}$. Note that if the parameter values are computed to be significantly outside the usual range, this could be taken as evidence of actual or impending device failure, thus providing a useful additional diagnostic.

This technique is clearly valuable for transmitters employing a time-delay based method of calculation, which is particularly vulnerable to voltage offset errors. However, to employ the technique it is necessary to carry out a superset of the Fourier-based phase calculations anyway, and hence to have a powerful processor. As a Fourier approach enjoys a variety of other advantages (see Henry et al, 2000) it is recommended that this be used.

\section{Hardware/Software Co-Design}

Another novel feature of the development of the coriolis prototype was the use of a hardware/software co-design technique known as hardware compilation (Henry, 1995c; Henry et al., 1996). In this approach, functionality to be carried out in hardware is described 
in Handel-C, a C-like language with parallel processing extensions. For prototyping, such functionality can be implemented within Field Programmable Gate Arrays (FPGAs), which can be reconfigured in milliseconds, while for a commercial implementation the same functionality might be enacted using one-time programmable components, an ASIC or even pulled back into a processor and executed as software, depending on commercial optimality.

In the coriolis prototype, a significant proportion of transmitter functionality was implemented using these tools, and in particular the entire amplitude control subsystem (Henry et al., 2000). This reads the sensor data and uses it to synthesise the drive waveform, a function carried out in analogue hardware within the commercial transmitter.

Ironically, much of the technology used to implement the prototype described in (Henry et al., 2000) is now obsolete. As well as using eight 3000-series Xilinx FPGAs, it was based on transputers, due to the legacy of prior research within the group. In parallel with the position manufacturers often experience, it became clear that migration to a new platform was essential. This proved straightforward: the code for the 3000 -series devices was ported into a single 4000-series FPGA, while the code for 4 transputers and a Power PC processor was migrated to an embedded PC-104 processor, running the VxWorks real-time operating system. Benefits of the migration include lower cost and size, and the provision of a Web interface to the new transmitter (Figure 11).

This migration to a new platform, albeit under research rather than commercial constraints, illustrates the potential benefits of a hardware-software co-design approach. However, commercialisation projects are now under way; these aim to maximise reuse of prototype design effort by embedding the same hardware compilation technology into commercial products. At this early stage it is not possible to report on the use of this technology within instrument manufacture, but this may form the basis of future publications.

\section{Summary}

Sensor validation (SEVA) is a technique which enables the analysis and quantification of measurement quality, specifically through the generation of on-line uncertainty. It includes quantification and where possible compensation for all defects, including manufacturing limitations and sensor faults. Its implementation, and any subsequent process of continuous instrument improvement, are rendered possible by the enormous advances in digital technology currently taking place. An overview of a new digital coriolis mass flow meter 
transmitter has been given, which demonstrates the benefits of redesign based on validation, and implementation using digital technology.

A detailed example has been provided of how computing power can be used to compensate for manufacturing limitations - in this case, the balance and zero voltage stability of the front-end circuitry and the low-cost audio ADC. The alternatives, namely, specifying more expensive components with better stability, or attempting production line calibration, restrict the manufacturer's choice of components (and/or manufacturing practice), which ultimately may lead to competitive disadvantage. A software solution is readily ported to a new platform and can readily accommodate advances in instrument fundamentals, whereas hardware or operational solutions (e.g. calibration) are expense and slow to re-engineer. 


\section{$\underline{\text { Appendix 1: Compensation Integrals and their approximations }}$}

Assuming the presence of up to three higher harmonics, the desired waveform for a sensor voltage $S V(t)$ is of the form:

$$
S V(t)=A_{1} \sin (\omega t)+A_{2} \sin (2 \omega t)+A_{3} \sin (3 \omega t)+A_{4} \sin (4 \omega t) \ldots \ldots
$$

where $A_{1}$ designates the amplitude of the fundamental frequency component and $A_{2}-A_{4}$ designate the amplitudes of the three harmonic components. However, in practice, the actual waveform is adulterated with zero offset $Z_{o}$ (which has a value close to zero) and mismatch between the negative and positive gains $G_{n}$ and $G_{p}$ (Fig. 5). Without any loss of generality, it can be assumed that $G_{p}$ equals unity and that $G_{n}$ is given by:

$$
G_{n}=1+\varepsilon_{G}
$$

where $\varepsilon_{G}$ represents the gain mismatch.

The technique assumes that the amplitudes $A_{i}$ and the frequency $\alpha$ are constant. This is justified because estimates of $Z_{o}$ and $\varepsilon_{G}$ are based on averages taken over many cycles(e.g., 10,000 interleaved cycles occurring in about 1 minute of operation). When implementing the technique, the algorithm tests for the presence of significant changes in frequency and amplitude to ensure the validity of the analysis. The presence of the higher harmonics suggests the use of Fourier techniques for extracting phase and amplitude information for specific harmonics. This entails integrating $S V(t)$ and multiplying by a modulating sine or cosine function.

The zero offset impacts the integral limits, as well as the functional form. Because there is a zero offset, the starting point for calculation of amplitude and phase will not be at the zero phase point of the periodic waveform $S V(t)$. For zero offset $Z_{o}$, the corresponding phase offset is, approximately,

$$
\varphi_{Z_{o}}=-\sin \left(\frac{Z_{0}}{A_{1}}\right) \ldots \ldots
$$

For small phase,

$$
\varphi_{Z_{o}}=-\frac{Z_{o}}{A_{1}}
$$

with corresponding time delay 


$$
t_{Z_{o}}=\frac{\varphi_{Z_{o}}}{\omega}
$$

The integrals are scaled (through pre-multiplication by a constant) so that the limiting value (i.e., as $Z_{o}$ and $\varepsilon_{G}$ approach zero) equals the amplitude of the relevant harmonic. The first two integrals of interest are:

$$
I_{1 P s}=\frac{2 \omega}{\pi} \int_{t_{Z_{o}}}^{\frac{\pi}{\pi}+t_{Z_{o}}}\left(S V(t)+Z_{o}\right) \sin \left[\omega\left(t-t_{Z_{o}}\right)\right] d t
$$

and

$$
I_{1 N \mathrm{~s}}=\frac{2 \omega}{\pi}\left(1+\varepsilon_{G}\right) \int_{\frac{\pi}{\omega}+t_{Z_{o}}}^{2 \frac{\pi}{\omega}+t_{Z_{o}}}\left(S V(t)+Z_{o}\right) \sin \left[\omega\left(t-t_{Z_{o}}\right)\right] d t
$$

These integrals represent what in practice is calculated during a normal Fourier analysis of the sensor voltage data. The subscript 1 indicates the first harmonic, $\mathrm{N}$ and $\mathrm{P}$ indicate, respectively, the negative or positive half cycle, and s and c indicate, respectively, whether a sine or a cosine modulating function has been used.

Strictly speaking, the mid-zero crossing point, and hence the corresponding integral limits, should be given by $\pi / \omega-t_{z o}$, rather than $\pi / \omega+t_{z o}$. However, the use of the exact mid-point rather than the exact zero crossing point leads to an easier analysis, and better numerical behaviour (due principally to errors in the location of the zero crossing point). The only error introduced by using the exact mid-point is that a small section of each of the above integrals is multiplied by the wrong gain ( 1 instead of $1+\varepsilon_{G}$ and vice versa). However, these errors are of order $Z_{o}^{2} \varepsilon_{G}$ and are considered negligible.

Using computer algebra and assuming small $Z_{o}$ and $\varepsilon_{G}$, first order estimates for the integrals may be derived as:

$$
I_{1 P s-\text { est }}=A_{1}+\frac{4}{\pi} Z_{o}\left[1+\frac{2}{3} \frac{A_{2}}{A_{1}}+\frac{4}{15} \frac{A_{4}}{A_{1}}\right]
$$

and

$$
I_{1 N s-e s t}=\left(1+\varepsilon_{G}\right)\left[A_{1}-\frac{4}{\pi} Z_{o}\left[1+\frac{2}{3} \frac{A_{2}}{A_{1}}+\frac{4}{15} \frac{A_{4}}{A_{1}}\right]\right]
$$

Useful related functions including the sum, difference, and ratio of the integrals and their estimates, may be determined. The sum of the integrals may be expressed as:

$$
\operatorname{Sum}_{1 \mathrm{~s}}=\left(I_{1 \mathrm{Ps}}+I_{1 \mathrm{Ns}}\right)
$$


while the sum of the estimates equals:

$$
\operatorname{Sum}_{1 s_{-} e s t}=A_{1}\left(2+\varepsilon_{G}\right)-\frac{4}{\pi} Z_{o} \varepsilon_{G}\left[1+\frac{2}{3} \frac{A_{2}}{A_{1}}+\frac{4}{15} \frac{A_{4}}{A_{1}}\right]
$$

Similarly, the difference of the integrals may be expressed as:

$$
\operatorname{Diff}_{1 \mathrm{~s}}=I_{1 \mathrm{Ps}}-I_{1 N \mathrm{~s}}
$$

while the difference of the estimates is:

$$
\operatorname{Diff}_{1 s}=-A_{1} \varepsilon_{G}+\frac{4}{\pi} Z_{o}\left(2+\varepsilon_{G}\right)\left[1+\frac{2}{3} \frac{A_{2}}{A_{1}}+\frac{4}{15} \frac{A_{4}}{A_{1}}\right]
$$

Finally, the ratio of the integrals is:

$$
\text { Ratio }_{1 s}=\frac{I_{1 P s}}{I_{1 N s}}
$$

while the ratio of the estimates is:

$$
\text { Ratio }_{1 s_{-} \text {est }}=\frac{1}{1+\varepsilon_{\mathrm{G}}}\left[1+\mathrm{Z}_{\mathrm{o}}\left[\frac{8}{15} \frac{15 \mathrm{~A}_{1}+10 \mathrm{~A}_{2}+4 \mathrm{~A}_{4}}{\pi \mathrm{A}_{1}^{2}}\right]\right]
$$

Corresponding cosine integrals are defined as:

$$
I_{1 P c}=\frac{2 \omega}{\pi} \int_{t_{z_{o}}}^{\frac{\pi}{\omega}+t_{Z_{o}}}\left(S V(t)+Z_{o}\right) \cos \left[\omega\left(t-t_{Z_{o}}\right)\right] d t
$$

and

$$
I_{1 N c}=\frac{2 \omega}{\pi}\left(1+\varepsilon_{G}\right) \quad \int_{\frac{\pi}{\omega}+t_{Z_{o}}}^{\frac{2 \pi}{\omega}+t_{Z_{o}}}\left(S V(t)+Z_{o}\right) \cos \left[\omega\left(t-t_{Z_{o}}\right)\right] d t
$$

with estimates:

$$
I_{1 P c_{-} e s t}=-Z_{o}+\frac{40 A_{2}+16 A_{4}}{15 \pi}
$$

and

$$
I_{1 N c_{-} \text {est }}=\left(1+\varepsilon_{G}\right)\left[Z_{o}+\frac{40 A_{2}+16 A_{4}}{15 \pi}\right] \ldots \ldots
$$


and sums:

and

$$
\operatorname{Sum}_{1 c}=I_{1 P c}+I_{1 N c}
$$

$$
\operatorname{Sum}_{1 c_{-} \text {est }}=\varepsilon_{G}\left[Z_{o}+\frac{40 A_{2}+16 A_{4}}{15 \pi}\right.
$$

Second harmonic integrals are:

$$
I_{2 P s}=\frac{2 \omega}{\pi} \quad \int_{T_{Z_{o}}}^{\frac{\pi}{\omega}+t_{Z_{o}}}\left(S V(t)+Z_{o}\right) \sin \left[2 \omega\left(t-t_{Z_{o}}\right)\right] d t
$$

and

$$
I_{2 N s}=\frac{2 \omega}{\pi}\left(1+\varepsilon_{G}\right) \int_{\frac{\pi}{\omega}+t_{Z_{o}}}^{\frac{2 \pi}{\omega}+t_{z_{o}}}\left(S V(t)+Z_{o}\right) \sin \left[2 \omega\left(t-t_{Z_{o}}\right)\right] d t
$$

with estimates:

$$
I_{2 P_{-} \text {est }}=A_{2}+\frac{8}{15 \pi} Z_{o}\left[-5+9 \frac{A_{3}}{A_{1}}\right]
$$

and

$$
I_{2 P s_{-} \text {est }}=\left(1+\varepsilon_{G}\right)\left[A_{2}-\frac{8}{15 \pi} Z_{o}\left[-5+9 \frac{A_{3}}{A_{1}}\right]\right]
$$

and sums:

$$
\operatorname{Sum}_{2 s}=I_{2 P s}+I_{2 N s}
$$

and

$$
\operatorname{Sum}_{2 \text { s }_{-} \text {est }}=A_{2}\left(2+\varepsilon_{G}\right)-\frac{8}{15 \pi} \varepsilon_{G} Z_{o}\left[-5+9 \frac{A_{3}}{A_{1}}\right]
$$

The integrals can be calculated numerically every cycle. As discussed in Appendix 2, the equations estimating the values of the integrals in terms of various amplitudes and the zero offset and gain values are rearranged to give estimates of the zero offset and gain terms based on the calculated integrals.

\section{Example}

The accuracy of the estimation equations may be illustrated with an example. In table 4, for each basic integral, three values are provided: the "true" value of the integral (calculated within Mathcad using Romberg integration), the value using the estimation equation, and the value calculated by the digital transmitter operating in simulation mode, using Simpson's rule for integration. 
Thus, for example, the value for $I_{1 P s}$ calculated according to:

$$
I_{1 P s}=\frac{2 \omega}{\pi} \int_{t_{Z_{o}}}^{\frac{\pi}{\omega}+t_{Z_{o}}}\left(S V(t)+Z_{o}\right) \sin \left[\omega\left(t-t_{Z_{o}}\right)\right] d t
$$

is 0.101353 , while the estimated value ( $\left.\mathrm{I}_{1 \text { Ps_est }}\right)$ calculated as:

$$
I_{1 P_{-} \_s t}=A_{1}+\frac{4}{\pi} Z_{o}\left[1+\frac{2}{3} \frac{A_{2}}{A_{1}}+\frac{4}{15} \frac{A_{4}}{A_{1}}\right]
$$

is 0.101358 . The value calculated using the digital transmitter in simulation mode is 0.101340. These calculations use the parameter values given in Table 3. Thus, at least for the particular values selected, the estimates given by the first order equations are accurate. As $Z_{o}$ and $\varepsilon_{G}$ approach zero, the errors in both the estimate and the simulation approach zero. 


\section{Appendix 2: Calculation of compensation factors}

The first order estimates for the integrals define a series of non-linear equations in terms of the amplitudes of the harmonics, the zero offset, and the gain mismatch. As the equations are non-linear, an exact solution is not readily available. However, an approximation followed by corrective iterations provides reasonable convergence with limited computational overhead.

Flowtube-specific ratios (varying with design and, perhaps, diameter) may be assumed for $A_{1}-A_{4}$. As such, no attempt is made to calculate all of the amplitudes $A_{1}-A_{4}$. Instead, only $A_{1}$ and $A_{2}$ are estimated using the integral equations defined above. Based on experience of the relative amplitudes, for a particular $25 \mathrm{~mm}$ flowtube design, $A_{3}$ is approximated as $A_{2} / 2$, and $A_{4}$ as $A_{2} / 10$.

The zero offset compensation technique has been implemented as follows. During each cycle, the controller calculates the integrals $I_{1 P s}, I_{1 N s}, I_{1 P c}, I_{1 N c}, I_{2 P s}, I_{2 N s}$ and related functions $\operatorname{sum}_{1 s}$, ratio $_{1 s}$, sum $_{1 c}$ and sum $_{2 s}$. This requires minimal additional calculation beyond the conventional Fourier calculations used to determine frequency, amplitude and phase, if these are used.

Every 10,000 cycles, the digital transmitter checks on the slope of the sensor voltage amplitude $A_{1}$. If the amplitude has been constant over the last minute, then the transmitter proceeds with calculations for zero offset and gain mismatch. This check may be extended to test for frequency stability.

To perform the calculations, the transmitter generates average values for the functions (e.g. sum $_{1 s}$ ) over the last 10,000 cycles. The transmitter then makes a first estimation of zero offset and gain mismatch as follows:

and

$$
Z_{o}=-S_{1 c} / 2
$$

$$
\varepsilon_{G}=1 / \text { Ratio }_{1 s}-1
$$

Using these values, the transmitter calculates an inverse gain factor $(\mathrm{k})$ and amplitude factor (amp_factor):

$$
k=1.0 /\left(1.0+0.5 * \varepsilon_{G}\right)
$$


and

$$
\text { amp_factor }=1+50 / 75 * \text { Sum }_{2 s} / \text { Sum }_{1}
$$

The transmitter uses the inverse gain factor and amplitude factor to make a first estimation of the amplitudes:

$$
A_{1}=k *\left[\operatorname{Sum}_{1 s} / 2+2 / \pi * Z_{o}^{*} \varepsilon_{G} * \text { amp_factor }\right]
$$

and

$$
A_{2}=k *\left[\operatorname{Sum}_{2 s} / 2-4 /\left(3^{*} \pi\right) * Z_{o}^{*} \varepsilon_{G}\right]
$$

The transmitter then improves the estimate by the following calculations, iterating as required:

$$
\begin{aligned}
& x_{1}=Z_{o} \\
& x_{2}=\varepsilon_{G} \\
& \varepsilon_{G}=\left[1+8 / \pi * x_{1} / A_{1} * a m p \text { factor }\right] / \text { Ratio }_{1 s}-1.0 \\
& Z_{o}=-\operatorname{Sum}_{1 c} / 2+x_{2} *\left(x_{1}+2.773 / \pi * A_{2}\right) / 2 \\
& A_{1}=k *\left[\operatorname{Sum}_{1 s} / 2+2 / \pi * x_{1} * x_{2} * a m p \text { factor }\right] \\
& A_{2}=k *\left[\operatorname{Sum}_{2 s} / 2-4 /(15 * \pi) * x_{1} * x_{2} *\left(5-4.5 * A_{2}\right)\right]
\end{aligned}
$$

The transmitter uses standard techniques to test for convergence of the values of $Z_{o}$ and $\varepsilon_{G}$. In practice the corrections are small after the first iteration, and experience suggests that three iterations are adequate.

Finally, the transmitter adjusts the raw data to eliminate $Z_{0}$ and $\varepsilon_{G}$ by adjusting the parameters used to convert the ADC channel output to voltage values. The transmitter then repeats the procedure over the next 10,000 cycles. Once zero offset and gain mismatch have been estimated for the first time, the functions (i.e., sum ${ }_{1 s}$ etc...) used in generating subsequent values for $Z_{o}$ and $\varepsilon_{G}$ are based on corrected data. Accordingly, these subsequent values for $Z_{o}$ and $\varepsilon_{G}$ reflect residual zero offset and gain mismatch, and are summed with previously generated values to produce the actual zero offset and gain mismatch. 


\section{$\underline{\text { References }}$}

- Cheesewright, R. and C. Clark (1998). The Effect of Flow Pulsations on Coriolis Mass Flow Meters. Journal of Fluids and Structures, 12, pp1025-1039.

- Cheesewright, R., C. Clark, D. Bisset (2000). The identification of external factors which influence the calibration of Coriolis massflow meters. Flow Measurement and Instrumentation 11, pp1-10.

- Clarke, D.W. (1998). Non-linear control of the oscillation amplitude of a Coriolis massflow meter. European Journal of Control. 4(3), pp196-207.

- Fuller, W.F. (1987). Direct Mass Flow Measurement using the Coriolis Principle. Australian Journal of Instrumentation and Control, 2(2), pp 6-9.

- Grumski, J.T. and R.A. Bajura (1984). Performance of a Coriolis type mass-flowmeter in the measuring of two-phase (air-liquid) mixtures. ASME Winter Annual Meeting, New Orleans, USA, 17, pp9-14.

- Henry, M.P. and D.W. Clarke (1993). The self-validating sensor: rationale, definitions and examples. Control Engineering Practice, 1(4), pp 585-610.

- Henry, M.P. (1994). Validating data from smart sensors. Control Engineering, 41(9), pp 63-66.

- Henry, M.P. (1995a). A self-validating Coriolis meter. Control Engineering, 42(6), pp 8186.

- Henry, M.P. (1995b). Fieldbus and sensor validation. IEE Computing and Control Journal, 6(6), pp 263-272.

- Henry, M.P. (1995c). Keynote Paper: Hardware compilation - a new technique for rapid prototyping of digital systems - applied to sensor validation. Control Engineering Practice, 3(7), 907-924.

- Henry, M.P., Archer, N., Atia, M.R.A., Bowles, J., Clarke, D.W., Fraher, P.M.A., Page, I., Randall, G., and Yang, J.C-Y. (1996). Programmable hardware architectures for sensor validation. Control Engineering Practice, 4(10), 1339-1354.

- Henry, M.P. (2000). Editorial, Special Issue on Intelligent and Self-Validating Sensors, IEE Computing and Control Engineering Journal, October 2000.

- Henry, M.P., D.W. Clarke, N. Archer, J. Bowles, M.J. Leahy, R.P. Liu, J. Vignos, F.B. Zhou (2000). A self-validating digital coriolis mass flow meter: an overview. Control Engineering Practice, 8(5), 487-506.

- SIRA (1998). The impact of Fieldbus and sensor fusion on flow measurement. DTI NMS Programme for Flow Measurement, Project OR9A Technical Awareness Studies.

- Wood, G.G. (2000). UK activities in measurement validation and data quality. IEE Computing and Control Engineering Journal, October 2000. 
- Yang, J.C-Y., and D.W. Clarke (1997). A self-validating thermocouple. IEE Transactions on Control Systems Technology, 5(2), 239-253. 


\section{Table Headings}

Table 1: Results of Fourier-based calculations, with frequency $82-95 \mathrm{~Hz}$, phase $0-4^{\circ}$, amplitude $0.3 \mathrm{~V}$

Table 2: Difference in batch total over 20 consecutive runs. Batch total $=200 \mathrm{~kg}$

Table 3: Parameter values used in simulation study results

Table 4: Comparison of intergration values using exact integration, approximation, and simulation. 
Table 1:

\begin{tabular}{cccc}
\hline & \multicolumn{3}{c}{ Standard Deviation } \\
& Frequency $(\mathrm{Hz})$ & Phase $\left({ }^{\circ}\right)$ & Amplitude (V) \\
\hline Signal Generator & $9.94 \mathrm{e}-4$ & $1.52 \mathrm{e}-3$ & $5.89 \mathrm{e}-6$ \\
Empty flowtube & $3.20 \mathrm{e}-4$ & $1.31 \mathrm{e}-3$ & $6.10 \mathrm{e}-6$ \\
0\% flow & $4.87 \mathrm{e}-4$ & $6.71 \mathrm{e}-3$ & $2.31 \mathrm{e}-5$ \\
10\% flow & $2.32 \mathrm{e}-3$ & $1.41 \mathrm{e}-2$ & $4.94 \mathrm{e}-4$ \\
$70 \%$ flow & $7.96 \mathrm{e}-3$ & $1.06 \mathrm{e}-1$ & $3.87 \mathrm{e}-3$ \\
\hline
\end{tabular}

Table 2

\begin{tabular}{cccc}
\hline $\begin{array}{c}\text { Flow } \\
\text { Rate } \\
(\mathrm{kg} / \mathrm{s})\end{array}$ & $\begin{array}{c}\text { Batch } \\
\text { Time } \\
(\mathrm{s})\end{array}$ & $\begin{array}{c}\text { Mean } \\
\text { Difference } \\
(\%)\end{array}$ & $\begin{array}{c}\text { Standard } \\
\text { Deviation } \\
(\%)\end{array}$ \\
\hline 4.0 & 50 & -0.0030 & 0.0102 \\
2.0 & 100 & 0.0887 & 0.0079 \\
1.0 & 200 & -0.2393 & 0.0053 \\
\hline
\end{tabular}

Table 3

\begin{tabular}{||l|l|l||}
\hline \multicolumn{1}{|c|}{ Parameter } & \multicolumn{1}{|c||}{ Value } & \multicolumn{1}{c|}{ Comment } \\
\hline$\omega$ & $160 \pi$ & This corresponds to frequency = 80Hz, a typical value. \\
\hline$A_{1}$ & 0.1 & This more typically is 0.3, but it could be smaller with, say, two-phase flow. \\
\hline$A_{2}$ & 0.01 & This more typically is 0.005, but it could be larger with two-phase flow. \\
\hline$A_{3}$ and $A_{4}$ & 0.0 & $\begin{array}{l}\text { The digital coriolis simulation mode only offers two harmonics, so these } \\
\text { higher harmonics are ignored. However, they are small }(<0.002) .\end{array}$ \\
\hline$Z_{o}$ & 0.001 & Experience suggests that this is a large value for zero offset. \\
\hline$\varepsilon_{G}$ & 0.001 & Experience suggests this is a large value for gain mismatch. \\
\hline
\end{tabular}

Table 4 


\begin{tabular}{|l|c|c|c||}
\hline \multicolumn{1}{|c|}{ Integral } & 'Exact' Value & Estimate & $\begin{array}{c}\text { Digital Coriolis } \\
\text { Simulation }\end{array}$ \\
\hline$I_{1 P s}$ & 0.101353 & 0.101358 & 0.101340 \\
\hline$I_{1 N s}$ & 0.098735 & 0.098740 & 0.098751 \\
\hline$I_{1 P c}$ & 0.007487 & 0.007488 & 0.007500 \\
\hline$I_{1 N c}$ & -0.009496 & -0.009498 & -0.009531 \\
\hline$I_{2 P s}$ & 0.009149 & 0.009151 & 0.009118 \\
\hline$I_{2 N s}$ & 0.010857 & 0.010859 & 0.010885 \\
\hline
\end{tabular}




\section{Figure Headings}

Fig. 1. Validation analysis leads to redesign.

Fig 2. Coriolis mass flow meter flowtube.

Fig. 3. Repeatability of the digital transmitter.

Fig. 4. Absolute accuracy of the digital transmitter.

Fig. 5. Voltage zero offset and gain imbalance of sensor signal waveforms.

Fig. 6. Voltage zero offset and gain imbalance for the two-channel Analogue Devices AD1879.

Fig. 7. Phase Difference calculated using time delay: A) positive zero-crossings only; B) negative zero-crossings only; C) all zero-crossings.

Fig. 8. Phase zero drift over 12 hours for time delay method (above) and Fourier method (below).

Fig. 9. Phase Difference calculated using time delay, using correction technique, from startup: A) positive zero-crossings only; B) negative zero-crossings only; C) all zero-crossings.

Fig. 10. Long term zero phase drift for Fourier and time delay method, using correction technique.

Fig. 11. Web-based interface for latest implementation of digital coriolis meter transmitter. 
Figures

Figure 1

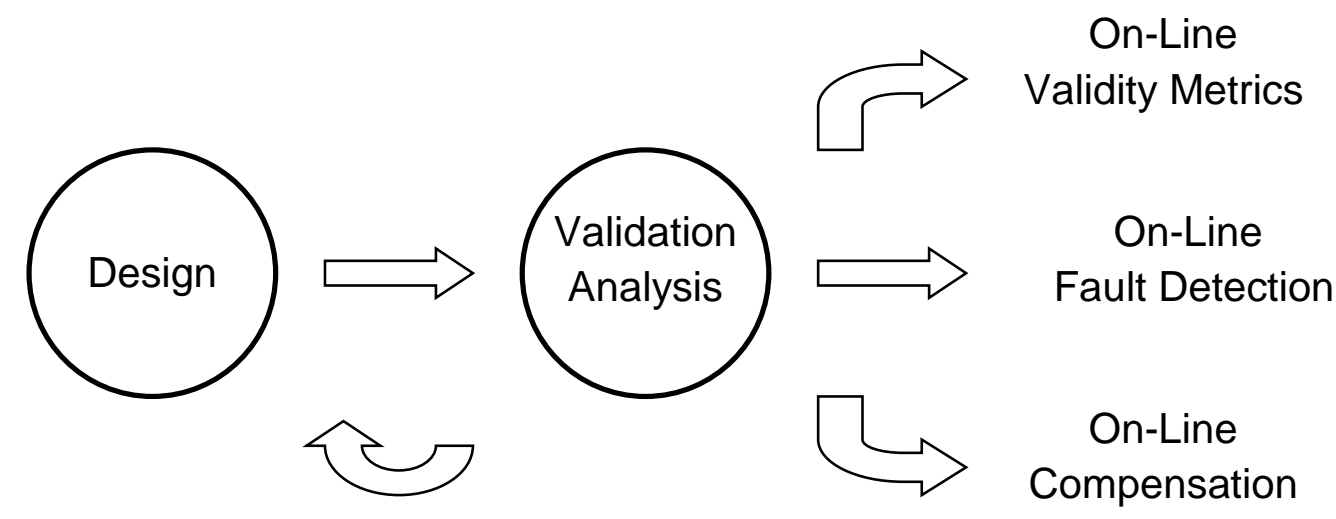

Criteria for redesign

Figure 2

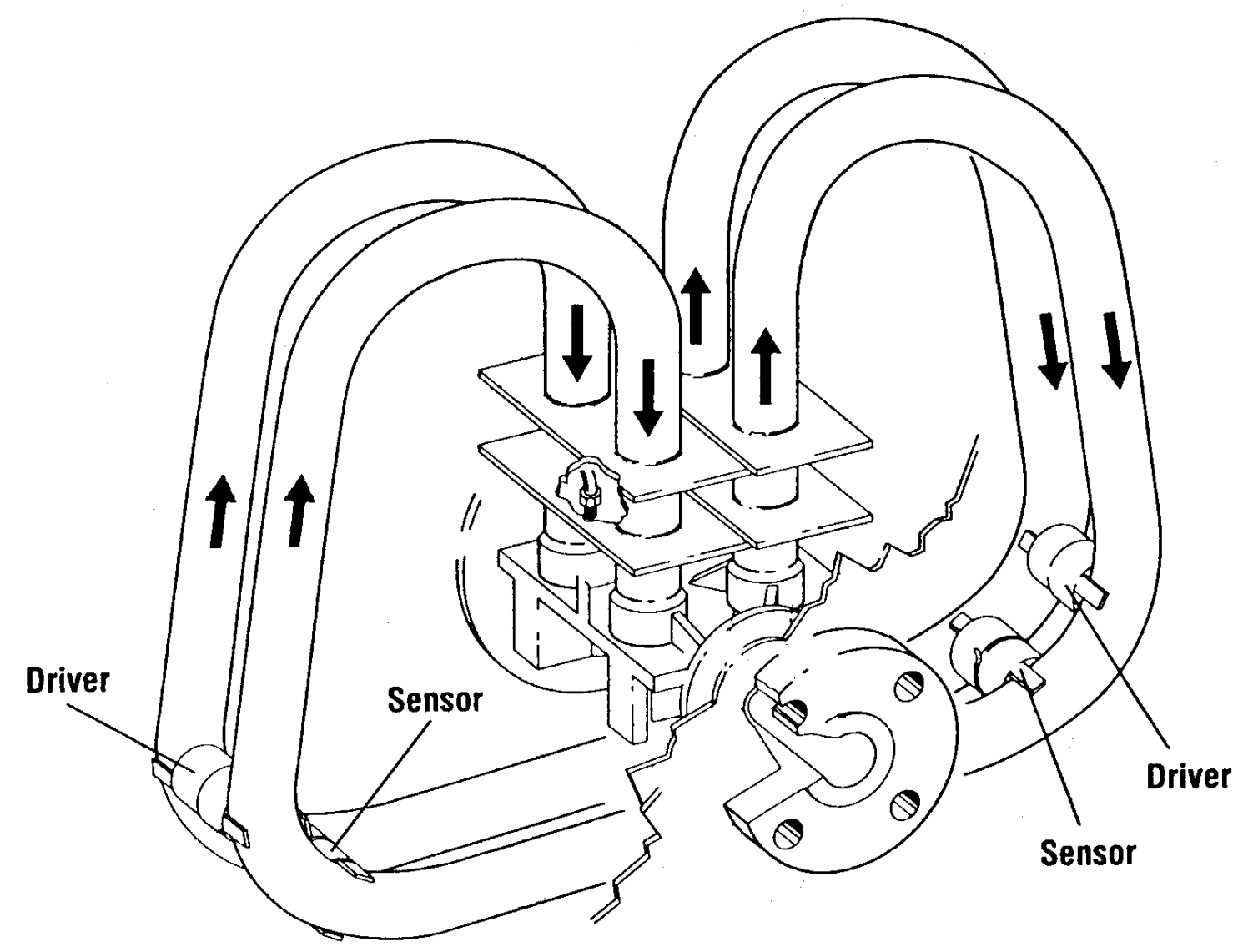


Figure 3

NORMAL PLOT FOR 2"-1" CORIOLIS NRPTELTY -

FLOW RATE $=1.0 \mathrm{~kg} / \mathrm{s}-3 . J U N 99$ - OXFORD DATA

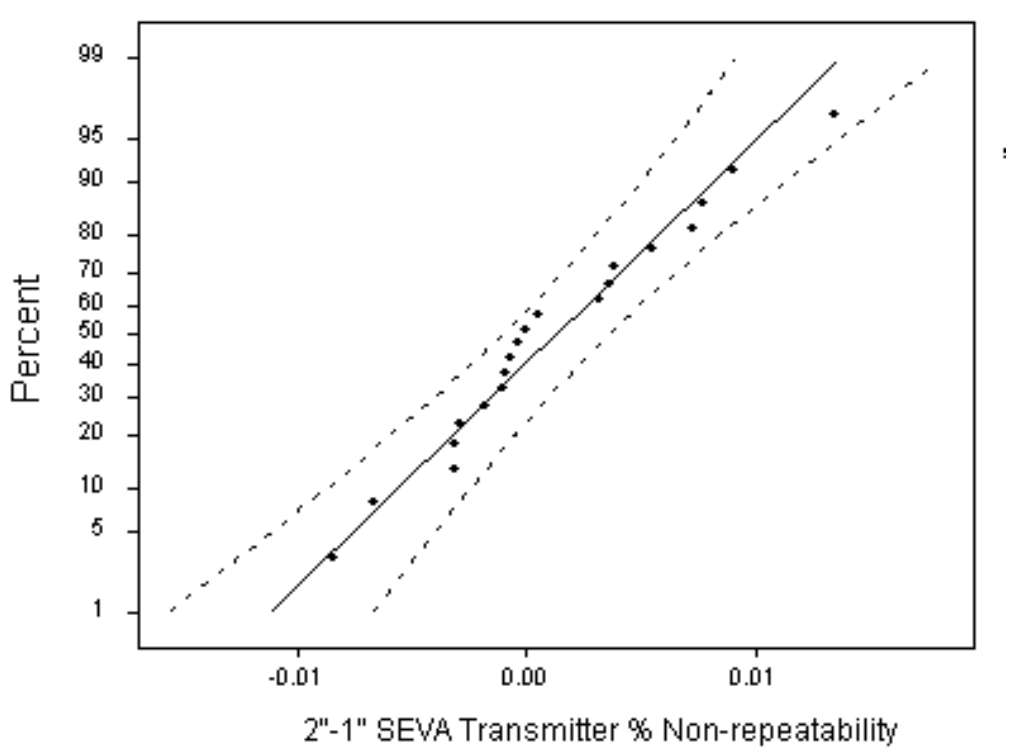

Figure 4

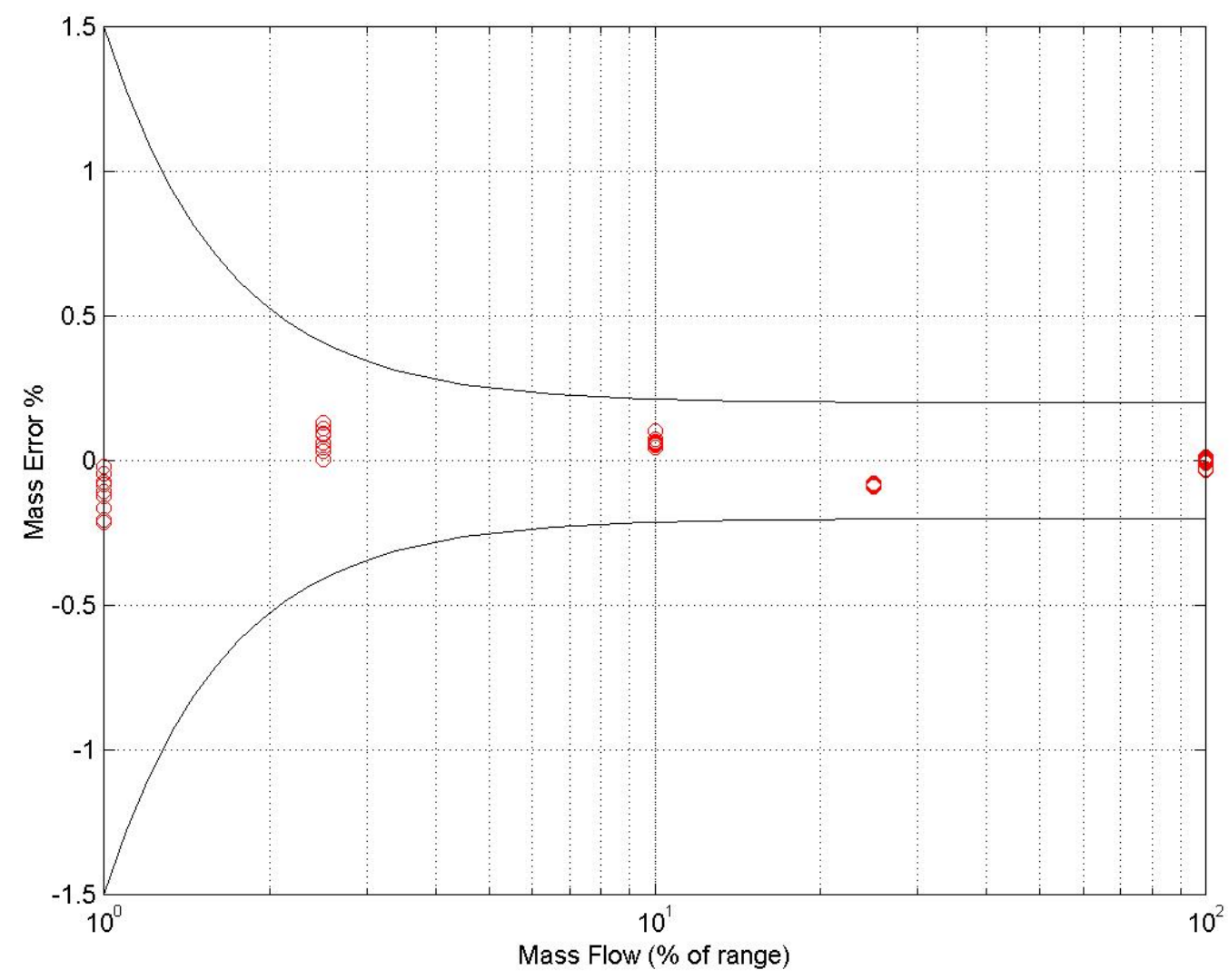


Figure 5.

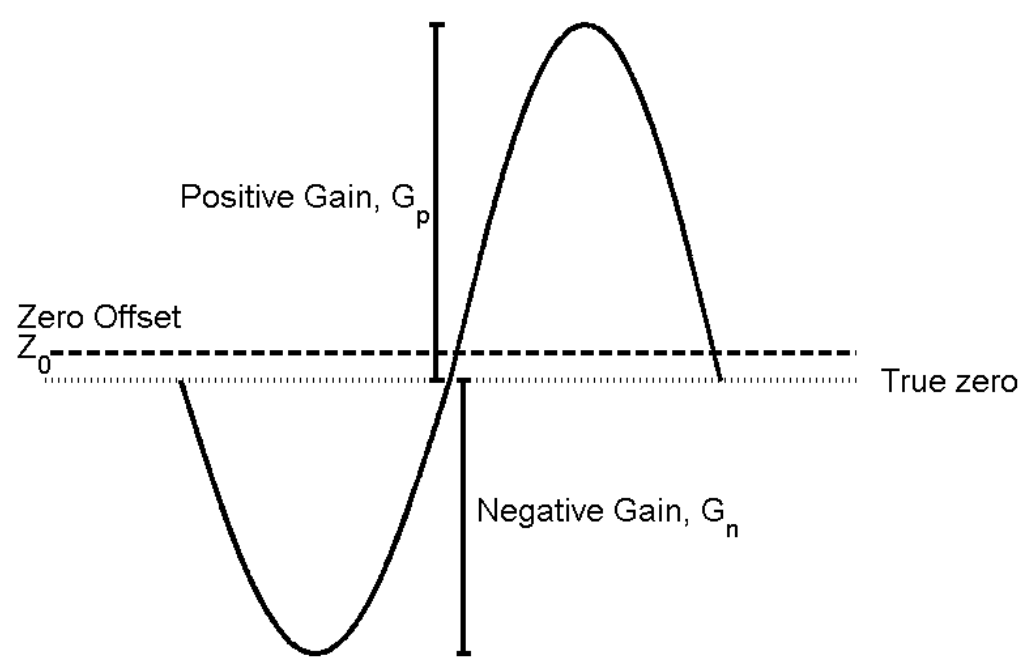

Figure 6.

Title:

plotcal.eps

Creator:

MATLAB, The Mathworks, Inc.

Preview.

This EPS picture was not saved

with a preview included in it.

Comment:

This EPS picture will print to a

PostScript printer, but not to

other types of printers. 
Fig. 7.
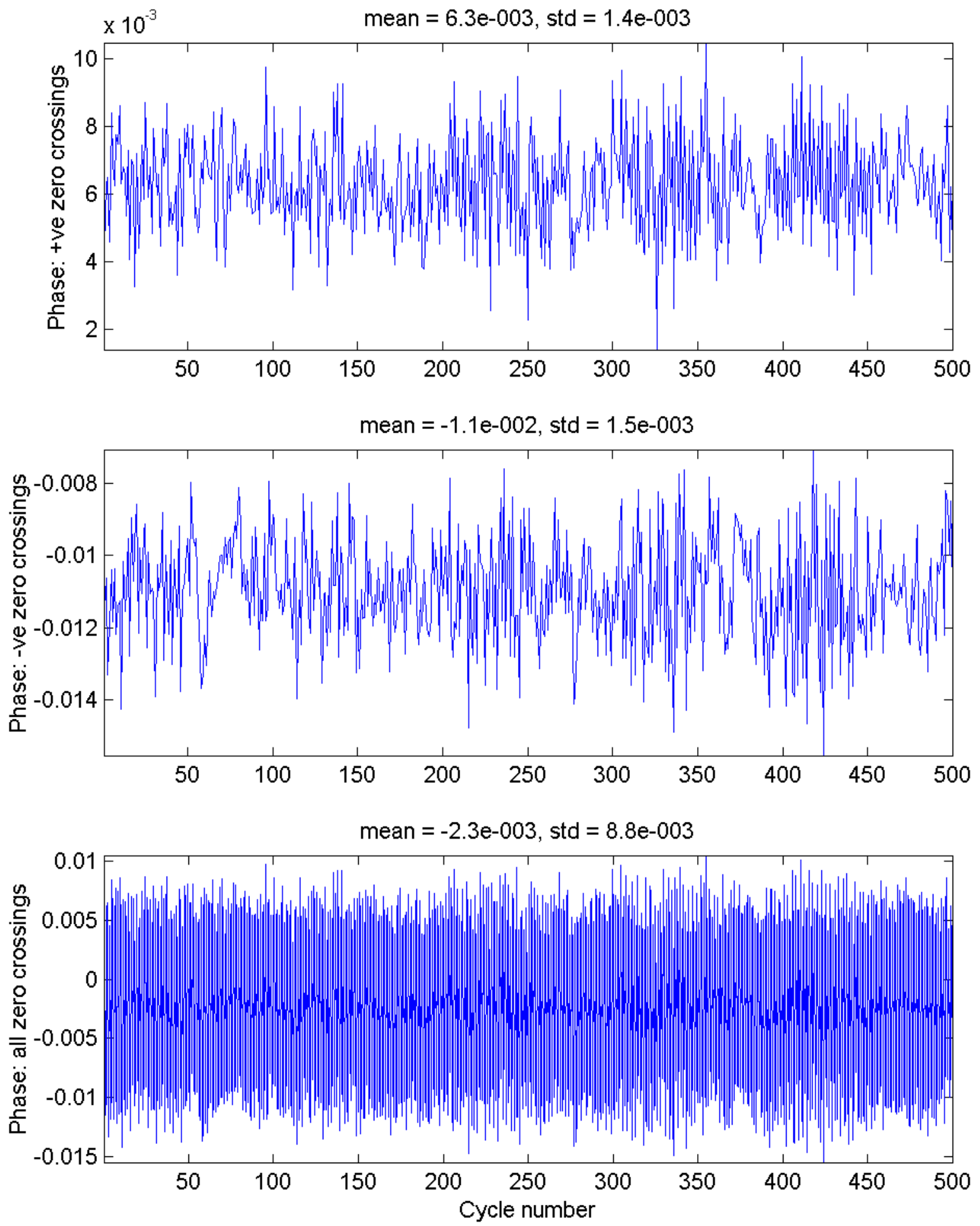

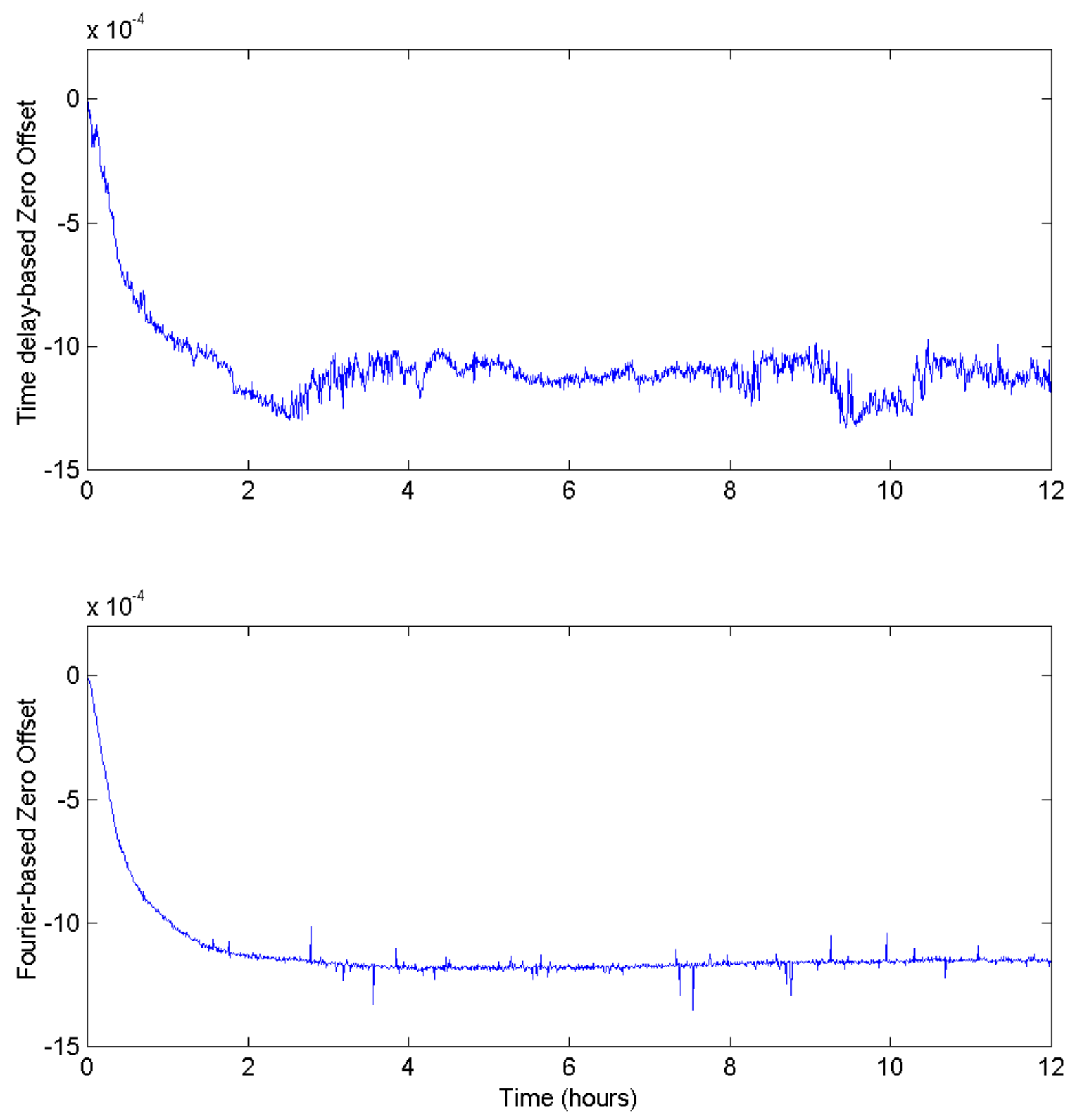

Fig. 8. 
Phase calculated using Time Delay technique
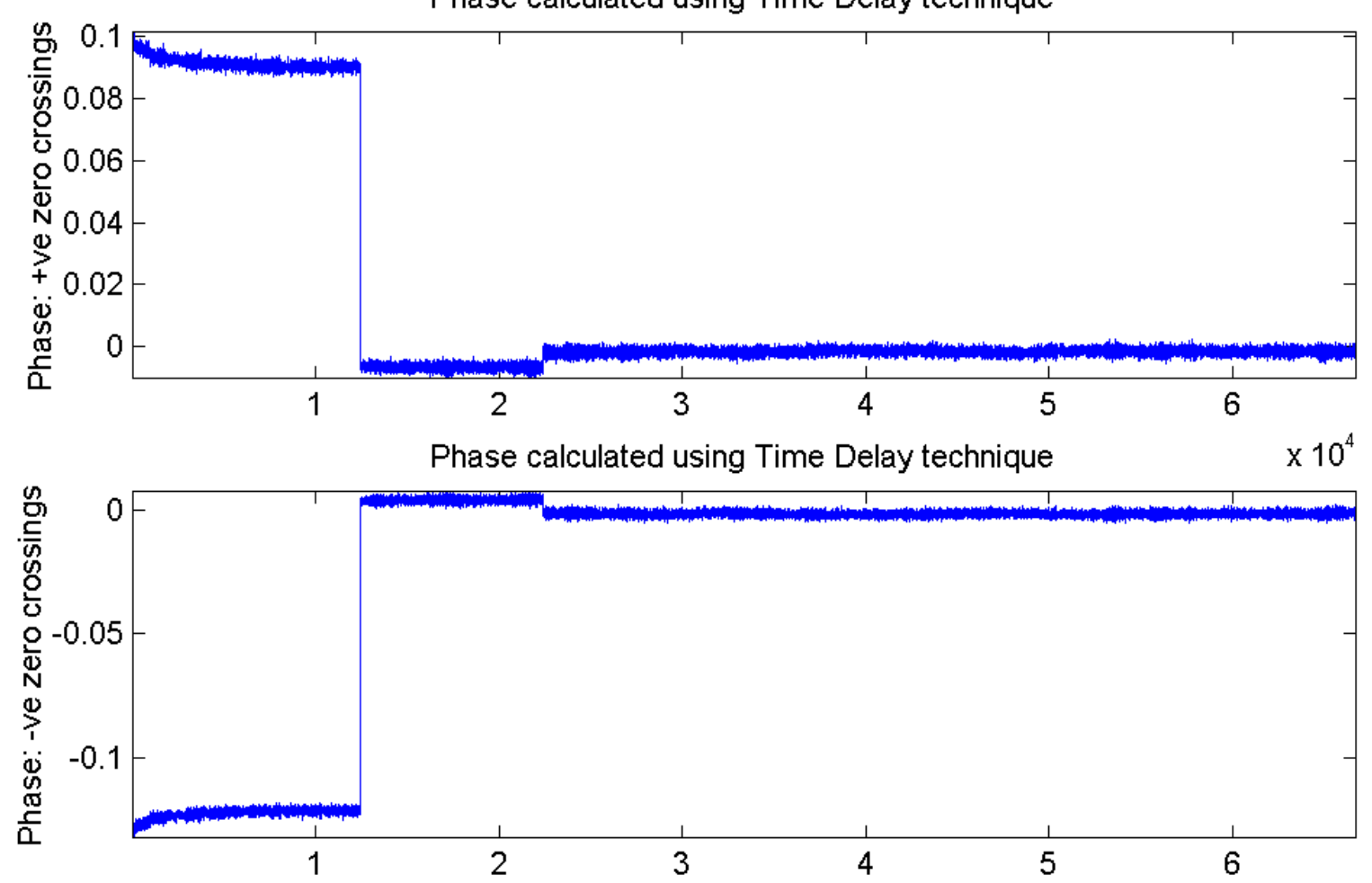

Phase calculated using Time Delay technique

$x 10^{4}$

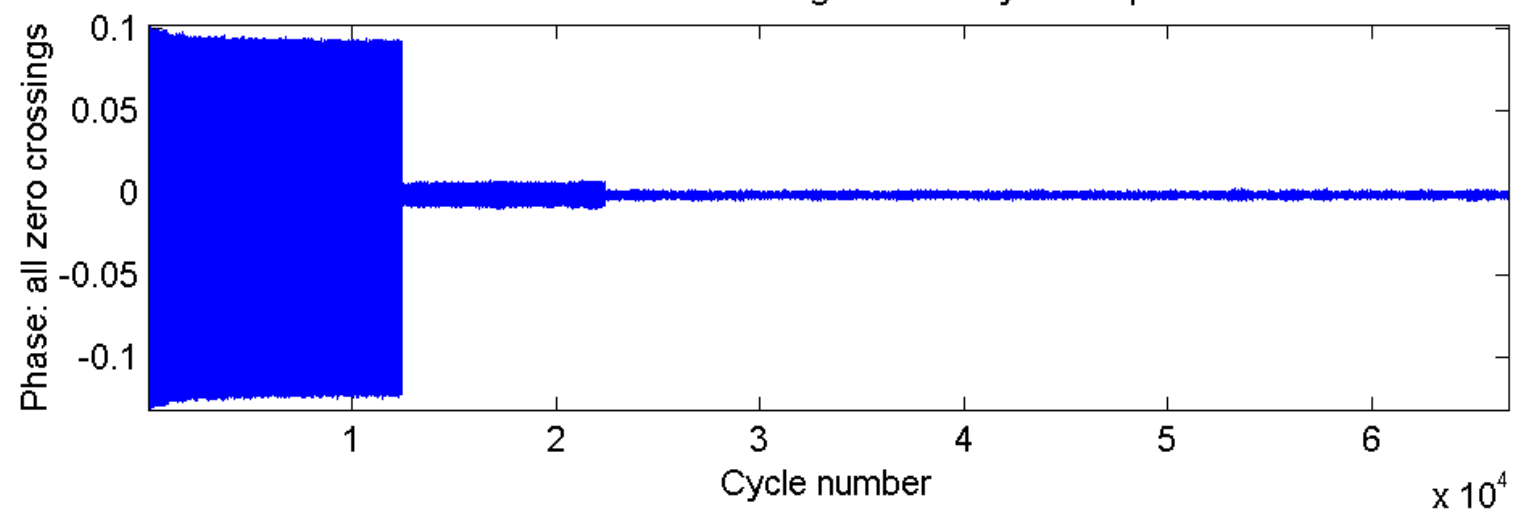

Figure 9. 


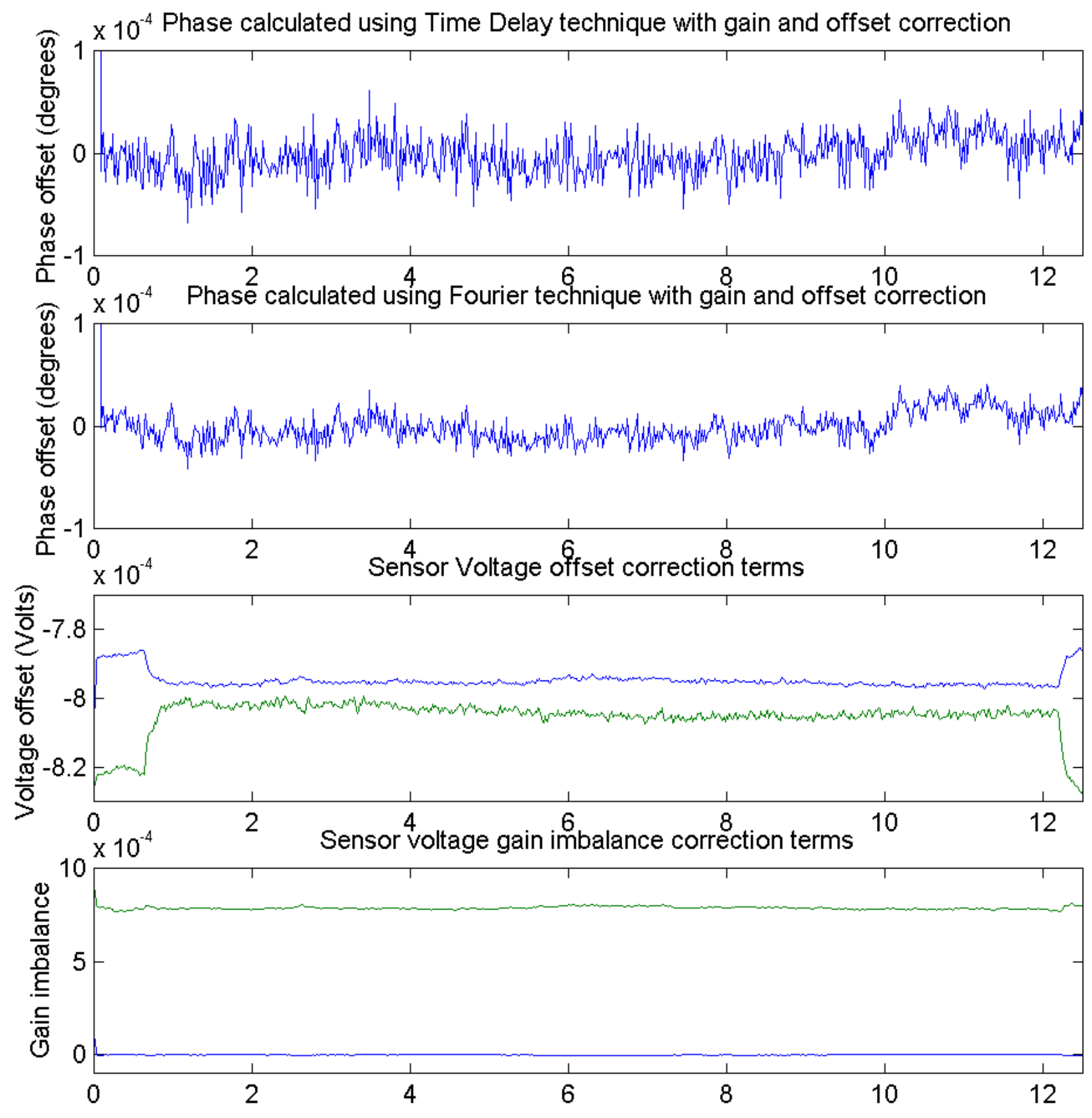

Figure 10. 
Figure 11.

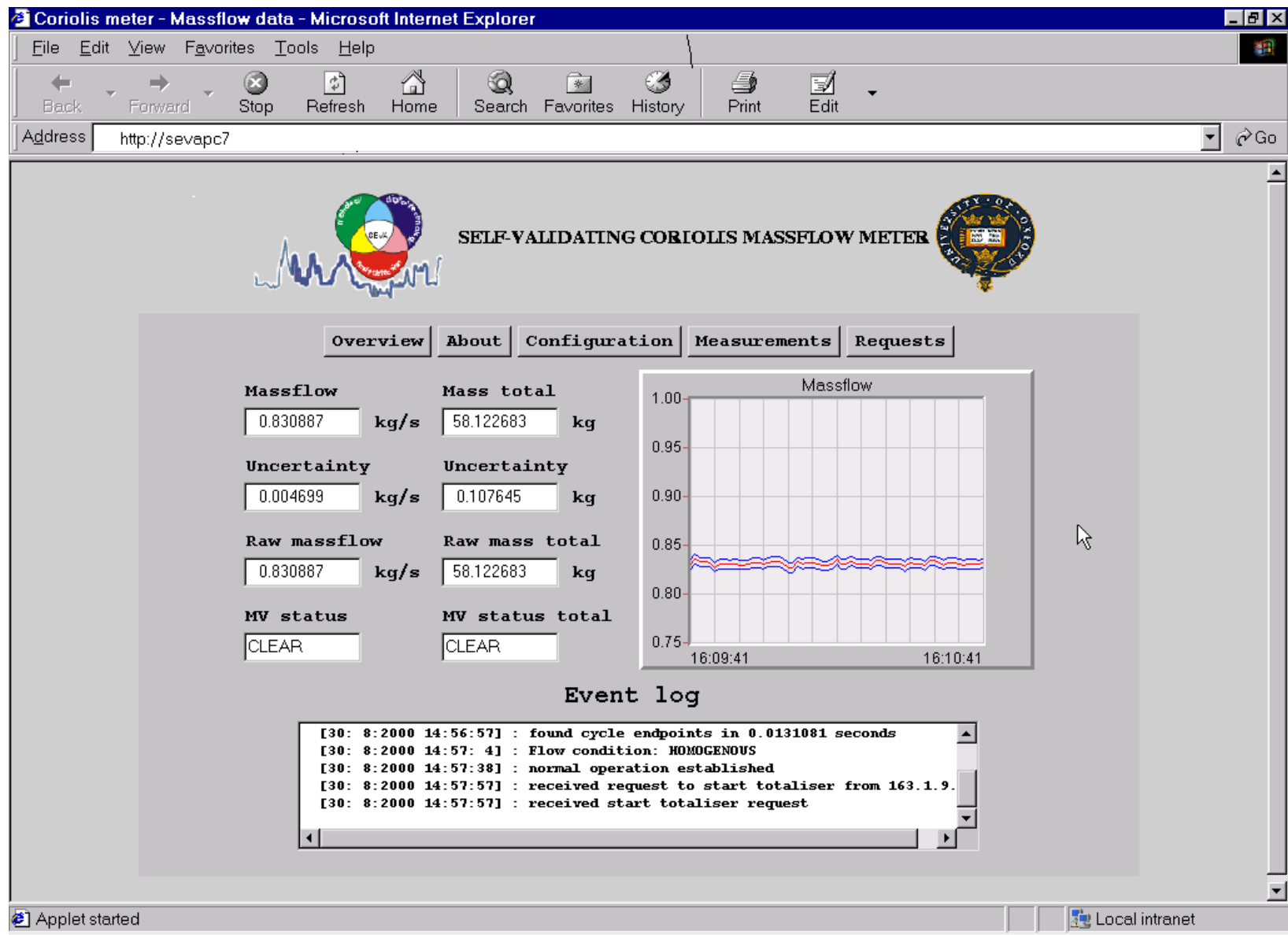

